\title{
Neuroticism, depression and anxiety in takotsubo cardiomyopathy
}

\author{
Thomas Emil Christensen 1,2* , Lia E. Bang1', Lene Holmvang ${ }^{1}$, Philip Hasbak², Andreas Kjær², Per Bech³ \\ and Søren Dinesen Østergaard ${ }^{4,5}$
}

\begin{abstract}
Background: Takotsubo cardiomypathy (TTC) causes acute reversible heart failure. Prior studies have indicated that the syndrome is associated with traits such as social inhibition, chronic psychological stress, and anxio-depressive disorders. The objective of this study was to further characterize key psychological/psychopathological traits of patients with TTC.

Methods: A survey of three groups was conducted: I) Female post-recovery TTC patients admitted between October $1^{\text {st }} 2009$ and December $10^{\text {th }}$ 2014, II) Age, gender and geographically matched ST-elevation myocardial infarction (STEMI) patients, and III) Age, gender and geographically matched individuals from the background population. The following questionnaires were used in the survey: the WHO-5 Well-Being Index, Eysenck's Neuroticism Scale, the Major Depression Inventory, and the anxiety subscale of Symptoms Checklist (SCL-90).

Results: In total, 173 of 230 invitees (75\%) participated in the study. In comparison to the background controls, TTC patients reported significantly less well-being, more neuroticism, more depression, and more anxiety. The levels of well-being, depression and neuroticism were comparable between TTC and STEMI patients, but the level of anxiety was higher in the TTC patients. There was a negative correlation between the time since TTC admission and the total scores on the psychopathology rating scales.

Conclusions: Patients with TTC reported significantly higher anxiety levels compared to both STEMI patients and background controls. However, unlike the STEMI patients, the TTC patients appeared to improve psychologically during the post-recovery phase. This may be a consolation for $\Pi C$ patients in acute psychological distress.
\end{abstract}

Keywords: Takotsubo cardiomyopathy, Well-being, Neuroticism, Depression, Anxiety

\section{Background}

Takotsubo cardiomypathy (TTC) is an increasingly recognized cause of acute heart failure mainly affecting elderly females [1]. The onset of TTC is often preceded by an emotional stressor [2], and catecholamine induced cardiomyopathy has been suggested as the underlying cause $[3,4]$. TTC patients typically have elevated cardiac biomarkers, electrocardiographic abnormalities including ST-elevations, but absence of a coronary culprit lesion on angiography. Left ventricle contractility is abnormal

\footnotetext{
* Correspondence: thomas.emil.christensen@regionh.dk

'Department of Cardiology, Copenhagen University Hospital, Rigshospitalet, Copenhagen, Denmark

${ }^{2}$ Department of Clinical Physiology, Nuclear Medicine \& PET and Cluster for Molecular Imaging, Copenhagen University Hospital, Rigshospitalet and University of Copenhagen, Copenhagen, Denmark

Full list of author information is available at the end of the article
}

with characteristic akinesia of the apical-midventricular segments, and basal normo- or hyperkinesia ('apical ballooning') [5]. Complete remission usually occurs within 1 month [6] and it was previously thought that TTC patients had an excellent prognosis. However, recent reports indicate a poor outcome similar to myocardial infarction [7].

It is well established that affective disorders constitute an independent risk factor for cardiovascular disease [8]. In the case of TTC, studies indicate that the syndrome is linked to psychopathological traits such as social inhibition, chronic psychological stress, and anxiodepressive disorders [9-12]. However, most studies have been conducted within a few months of initial admission, and thus recent life events related to the onset of TTC may have confounded the results. Therefore, the 
aim of this study was to characterize key psychological/ psychopathological traits in a large, carefully diagnosed cohort of post-recovery TTC patients using well-validated psychometric tools. For comparison, we included one group of patients with previous ST elevation myocardial infarction (STEMI), and one group of background population controls. We hypothesized that specific psychopathological traits were more pronounced in the patients with TTC compared to the STEMI patients and the individuals in the background control group.

\section{Method}

\section{Participants}

A total of 45 TTC patients were prospectively recruited among female patients admitted at the Department of Cardiology, Copenhagen University Hospital from October $1^{\text {st }} 2009$ to December $10^{\text {th }} 2014$. Inclusion criteria were (1) acute onset of symptoms, (2) no culprit lesion on coronary angiography, (3) typical 'apical ballooning', (4) elevated cardiac biomarkers, and (5) normalized left ventricle systolic function on follow-up echocardiography. The STEMI patients were 95 age- and geographically matched females with previous one-vessel disease and STEMI that had received primary coronary intervention at Copenhagen University Hospital within the TTC inclusion period. The background controls were 90 age- and geographically matched females randomly selected from the Danish Civil Registration Registry [13].

\section{Survey}

The survey was conducted between February and April 2015. A questionnaire for self-reporting was mailed to the invitees' home addresses, and a maximum of two reminders were sent by letter if invitees did not respond to the first questionnaire. The questionnaire consisted of the following self-rating scales: The 5-item WHO-5 Well-Being Index (WHO-5) [14], the 23-item Eysenck's Neuroticism Scale (ENS) [15, 16], the 10-item Major Depression Inventory (MDI) [17], and the 8-item anxiety subscale of Hopkin's Symptoms Checklist (ASS) [18]. These self-rating scales are well-validated measures of well-being, neuroticism, depression, and anxiety, respectively. Not all questionnaires were fully filled out by the participants. If $>20 \%$ of the item scores on one of the four self-rating scales were missing, this entire scale was excluded from further analysis. If $\leq 20 \%$ of the item scores were missing on a self-rating scale, the missing scores were replaced by the mean value of the completed item scores of this scale.

\section{Statistics}

Unless stated otherwise, results are presented as median (interquartile range). The total score for each of the four rating scales was compared pair-wise between groups by means of the Wilcoxon-Mann-Whitney test. Correlations between rating scale total scores and age, and time since admission respectively, were tested by Spearman rank ( $r$ ) correlation analysis. Both the Wilcoxon-MannWhitney test and the $r$ correlation analyses were performed using SAS Statistical software (version 9.0) with the level of significance set at two-tailed $p<0.05$. As we intended to use the total scores of each of the four selfrating scales as measures for overall well-being (WHO5) or syndrome severity (END, MDI, and ASS), we tested the scalability of these scales. Scalability is present when each of the individual item scores of a scale contributes unique information regarding the dimension of interest. Only when this is the case can the individual item scores be summed to a meaningful total score [19]. The analysis of scalability was performed using the Mokken non-parametric item response theory model, where the degree of scalability is expressed by the coefficient of homogeneity. A coefficient of 0.40 or higher indicates acceptable scalability [20]. The Mokken analysis was performed with the dedicated MSP 3.0 software [21].

\section{Ethics}

The study was approved by the regional ethics committee, and all participants provided written consent.

\section{Results}

The flow of participants in the survey is depicted in Fig. 1. In total, 173 of 230 (75\%) invitees, 40 TTC patients ( $89 \%$ of invitees), 71 STEMI patients (75\% of invitees) and 62 background controls (69\% of invitees), participated in the study. Age in years was $70(64 ; 76)$ for the TTC patients, $72(64 ; 77)$ for the STEMI patients, and $67(64 ; 70)$ for the background controls. The age of the TTC and the STEMI patients was comparable $(p=$ $0.79)$, whereas the background controls were significantly younger than both the TTC patients $(p=0.02)$, and the STEMI patients $(p=0.003)$. The time from initial admission to the survey was $24(8 ; 36)$ months and 26 (22; 32) months for TTC and STEMI patients, respectively, with no statistically significant difference between the two groups $(p=0.70)$. The Mokken analysis showed that all of the self-rating scales included in the survey had acceptable coefficients of homogeneity $(\geq 0.40)$ : WHO-5 0.65, ENS 0.40, ASS 0.66, and MDI 0.53 . This entails that the total scores of the scales are valid (scalable) measures of the psychological/psychopathological dimensions being investigated [19].

The results of the survey are presented in Fig. 2. Wellbeing and depression scores were comparable between the TTC and STEMI groups (WHO-5: 64 (40; 80) vs. 68 (52; 80), $p=0.57$. MDI: $8(3 ; 16)$ vs. $7(3 ; 14), p=0.72)$. ENS score tended to be higher in the TTC group (ENS: $9(3 ; 13)$ vs. $5(2 ; 11), p=0.08)$, but the difference was 


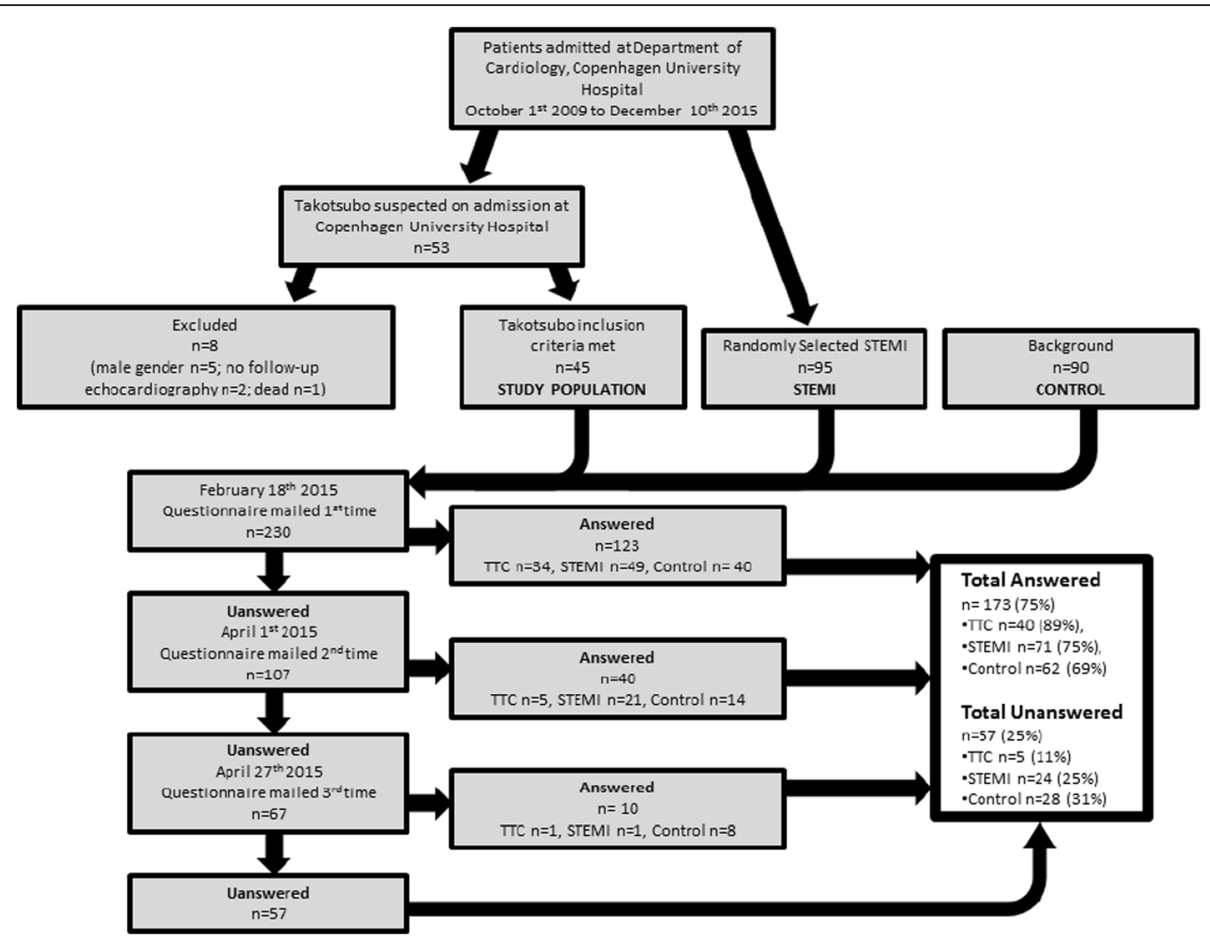

Fig. 1 Flow chart depicting study inclusion

not statistically significant. The anxiety score was significantly higher in the TTC than the STEMI group (ASS: $2(1 ; 7)$ vs. $1(0 ; 3), p=0.007)$. When compared to the control group, TTC patients reported significantly less well-being (WHO-5: $76(68 ; 84)$ vs. $64(40 ; 80), p=0.02)$, higher neuroticism (ENS: 3 (0; 9) vs. 9 (3; 13), $p=0.0002$ ), more depression (MDI: $4(1 ; 9)$ vs. $8.0(3 ; 16), p=0.007)$, and more anxiety (ASS: $1(0 ; 2)$ vs. $2(1 ; 7), p=0.0001)$. Compared to the background controls, the STEMI patients reported less well-being (WHO-5: 76 [68; 84] vs.

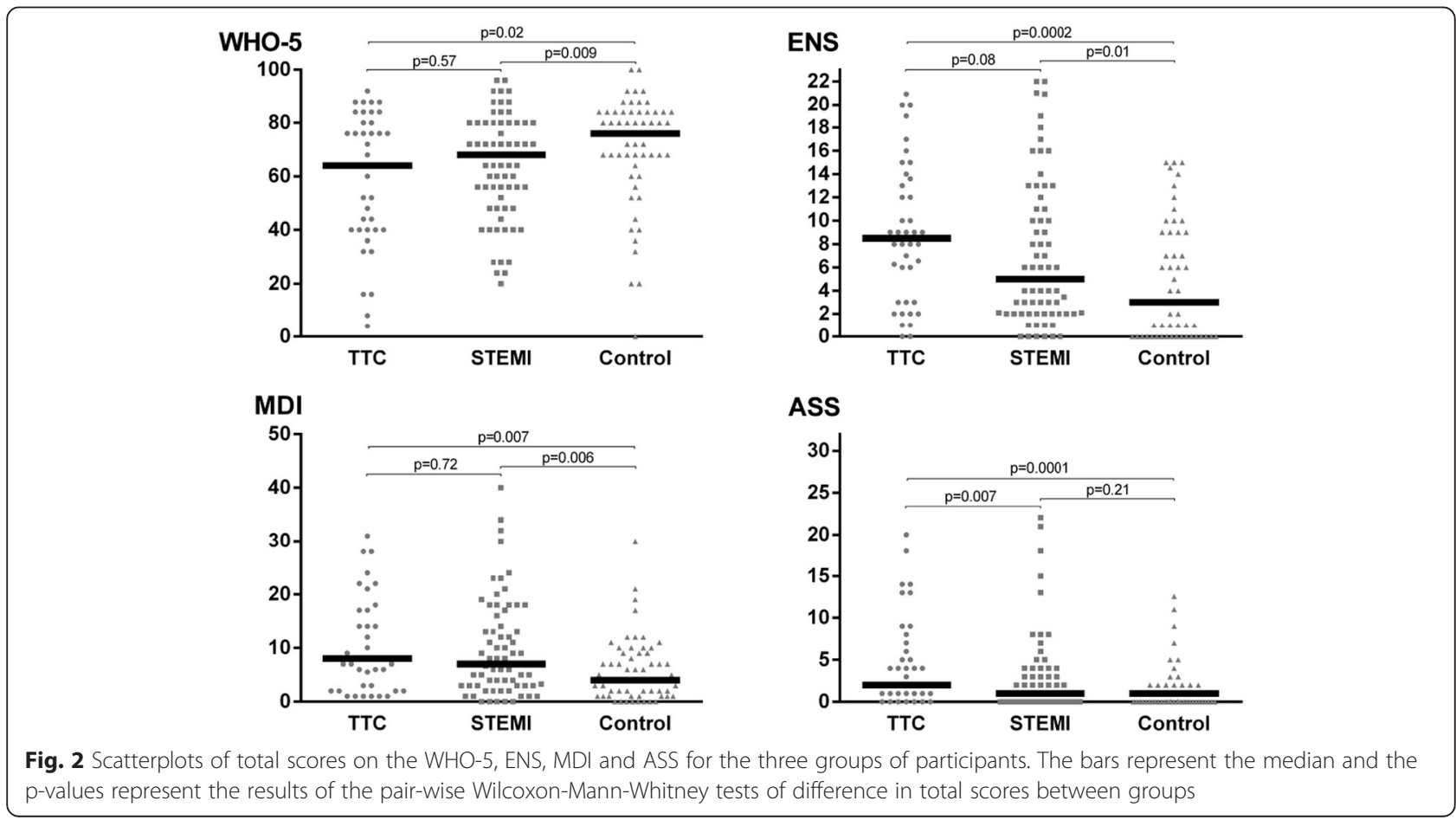


68 [52; 80], $p=0.009$ ), more neuroticism (ENS: 3 [0; 9] vs. $5[2 ; 11], p=0.01$ ), more depression (MDI: 4 [1; 9] vs. 7 [3; 14 ], $p=0.006$ ), whereas the level of anxiety was similar (ASS: $1[0 ; 2]$ vs. $1[0 ; 3], p=0.21$ ). There was no significant correlation between age and any of the total scores on the four self-rating scales (Age and WHO-5: $r=0.06, p=$ 0.46, $n=173$; Age and ENS: $r=0.01, p=0.94, n=169$;
Age and MDI: $r=0.01, p=0.94, n=173$; Age and ASS: $r=-0.01, p=0.93, n=171$ ).

In Fig. 3, the time between admission and survey participation is plotted against the total scores of the WHO-5, ENS, MDI and ASS for the TTC and STEMI patients, respectively. For the STEMI patients, there was no significant correlation between time and total scores

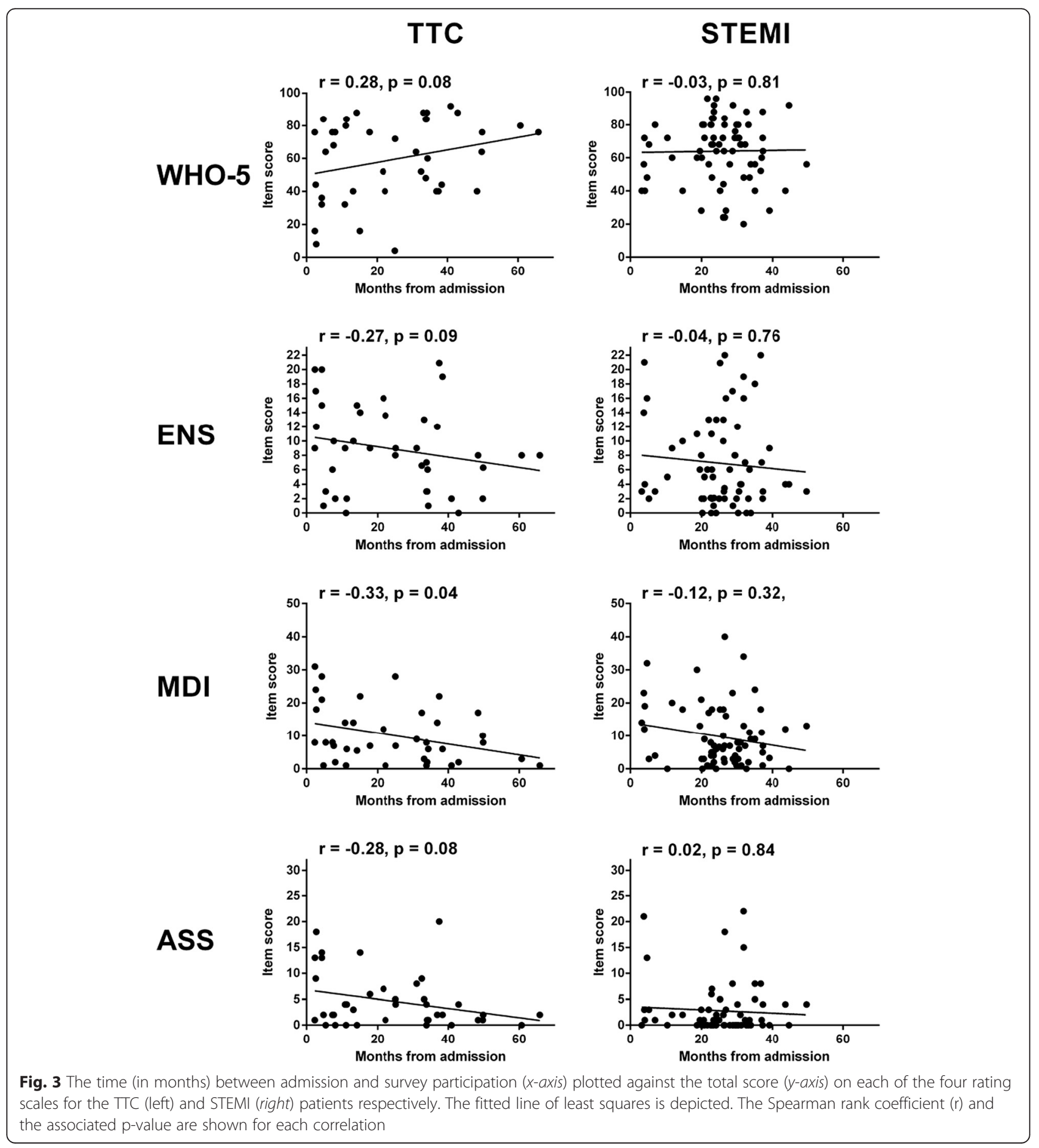


(Time and WHO-5: $r=-0.03, p=0.81, n=70$; Time and ENS: $r=-0.04, p=0.76, n=69$; Time and MDI: $r=-0.12$, $p=0.32, n=70$; Time and ASS: $r=0.02, p=0.84, n=68$ ). Conversely, the same correlations were either significant or near-significant $(p<0.1)$ for the TTC patients (Time and WHO-5: $r=0.28, p=0.08, n=40$; Time and ENS: $r=-0.27, p=0.09, n=40$; Time and MDI: $r=-0.33$, $p=0.04, n=40$; Time and ASS: $r=-0.28, p=0.08, n=40$ ), which is indicative of psychological improvement in the post-recovery phase of TTC.

\section{Discussion}

We examined four psychological/psychopathological dimensions in post-recovery female TTC patients, ageand geographically matched female STEMI patients and age- and geographically matched females from the background population. As expected, the results showed that TTC patients reported significantly less well-being, more neuroticism, more depression, and more anxiety than the background controls. The level of well-being, depression and neuroticism in TTC patients was comparable to that of the STEMI patients, but the TTC patients were significantly more anxious than the STEMI patients. These findings are consistent with previous studies of the psychological/psychopathological profile of patients with TTC. Specifically, Compare et al. compared the prevalence of Type D personality in TTC patients with an emotional trigger to TTC patients without an emotional trigger and to STEMI patients. Type D personality is characterized by negative affect and social inhibition, and the frequency of Type D personality was higher in the TTC patients with an emotional trigger than in the two other groups [9]. Similarly, Delmas et al. showed that anxio-depressive disorders and chronic psychological stress was common in TTC patients and occurred more often than in patients with coronary disease [10]. Finally, Kastaun et al. found that TTC patients showed impaired cortisol release in response to stress, and were more anxious, worried and socially inhibited when compared to STEMI and healthy controls [11].

In contrast to STEMI, TTC leaves no permanent myocardial damage [22]. Thus, TTC patients are generally not considered to be chronically ill. Despite this fact, both the present study and the literature in general indicate that TTC is associated with more pronounced psychopathology compared to chronic ischemic heart disease. However, the results of our study also indicated that TTC patients, as opposed to STEMI patients, tend to improve psychologically during the post-recovery phase. To our knowledge, this is a novel finding, which may be a consolation for TTC patients in psychological distress in the acute phase.

Limitations to this study warrant a mention. First and foremost, since our survey was conducted post-TTC, we were not able to investigate the direction of causality of the psychopathology-TTC association, i.e., whether individuals develop TTC because they are psychologically vulnerable, or whether they develop psychopathology because of TTC? Since TTC is believed to be caused by sympathetic hyperactivity [3, 4] and patients with anxiety disorders show increased sympathetic reactivity in response to stress $[23,24]$, it seems plausible that the direction of causality goes from psychopathology to TTC. This hypothesis is supported by a small retroperspective study by Summers et al. [12] in which past medical records of TTC patients were reviewed and compared to those of STEMI patients and background controls. Compared to the two other groups, the TTC patients had a much higher prevalence of anxiety disorders in their pre-cardiac-illness medical history. However, definitive prospective studies of this association are lacking from the literature. This is probably due to the fact that with the relatively low incidence of TTC (approximately $2 \%$ of patients admitted on suspicion of acute coronary syndrome [1]), it will require extremely large surveys with very long follow-up to obtain pre-morbid psychological/ psychopathological data on a sufficient number of TTC patients. Consistently, the small sample size is also a limitation of the present retrospective study.

Another limitation of our study is the risk of selfselection-bias as not all invitees completed the survey. Indeed, the age of the controls was slightly lower than that of the TTC and STEMI patients, but the Spearman rank correlation analysis revealed no significant correlation between age and rating scale total scores. It therefore seems unlikely that the lower age of the background controls has biased the results. Since many baseline data were not collected, we cannot exclude that confounders such as differences in educational or income level between groups have contributed to our results.

\section{Conclusion}

In conclusion, we found that post-recovery TTC patients reported levels of well-being, neuroticism, and depression that are comparable to those of STEMI patients. However, TTC patients reported higher levels of anxiety than STEMI patients. In contrast to STEMI patients, the TTC patients appear to improve psychologically during the post-recovery phase. This may be a consolation for patients in psychological distress in the acute phase of TTC.

\section{Abbreviations}

ASS, 8-item anxiety subscale of Hopkin's Symptoms Checklist; ENS, 23-item Eysenck's Neuroticism Scale; MDI, 10-item Major Depression Inventory; STEMI, STelevation myocardial infarction; TTC, Takotsubo 
cardiomyopathy; WHO-5, World Health Organization5 Well-Being Index.

\section{Acknowledgements}

The authors are grateful to the study participants.

\section{Funding}

Søren Dinesen Østergaard was funded by a grant from the Lundbeck Foundation. The remaining authors were funded by their respective departments.

\section{Availability of data and materials}

The dataset without direct or indirect identifiers can be obtained from the corresponding author upon request.

\section{Authors' contributions}

All authors contributed to the design and conduct of this study. The manuscript was drafted by Thomas Emil Christensen and Søren Dinesen Østergaard, and was critically revised by the remaining authors. The final version of the manuscript was approved by all authors prior to submission.

\section{Competing interests}

The authors declare that they have no competing interests.

\section{Consent for publication}

Not applicable.

\section{Ethics}

The study was approved by the Regional Committee on Health Research Ethics (protocol\# H-4-2010-054), and by the Danish data protection agency (File\# 30-1314). All participants provided written consent.

\section{Author details}

'Department of Cardiology, Copenhagen University Hospital, Rigshospitalet, Copenhagen, Denmark. ${ }^{2}$ Department of Clinical Physiology, Nuclear Medicine \& PET and Cluster for Molecular Imaging, Copenhagen University Hospital, Rigshospitalet and University of Copenhagen, Copenhagen, Denmark. ${ }^{3}$ Psychiatric Research Unit, Psychiatric Center North Zealand, Copenhagen University Hospital, Hillerød, Denmark. ${ }^{4}$ Department of Clinical Medicine, Aarhus University Hospital, Aarhus, Denmark. ${ }^{5}$ Department P Research, Aarhus University Hospital, Risskov, Denmark.

\section{Received: 27 November 2015 Accepted: 13 May 2016}

Published online: 31 May 2016

\section{References}

1. Akashi YJ, Nef HM, Lyon AR. Epidemiology and pathophysiology of Takotsubo syndrome. Nat Rev Cardiol. 2015;12:387-97.

2. Akashi YJ, Goldstein DS, Barbaro G, Ueyama T. Takotsubo cardiomyopathy: a new form of acute, reversible heart failure. Circulation. 2008:118:2754-62.

3. Wittstein IS. Stress cardiomyopathy: a syndrome of catecholamine-mediated myocardial stunning? Cell Mol Neurobiol. 2012;32:847-57.

4. Lyon AR, Rees PS, Prasad S, Poole-Wilson PA, Harding SE. Stress (Takotsubo) cardiomyopathy-a novel pathophysiological hypothesis to explain catecholamine-induced acute myocardial stunning. Nat Clin Pract Cardiovasc Med. 2008;5:22-9.

5. Madhavan M, Prasad A. Proposed Mayo Clinic criteria for the diagnosis of Tako-Tsubo cardiomyopathy and long-term prognosis. Herz. 2010;35:240-3.

6. Ako J, Sudhir K, Farouque HM, Honda Y, Fitzgerald PJ. Transient left ventricular dysfunction under severe stress: brain-heart relationship revisited. Am J Med. 2006;119:10-7.

7. Redfors $B$, Vedad R, Angeras $\mathrm{O}$, et al. Mortality in takotsubo syndrome is similar to mortality in myocardial infarction - A report from the SWEDEHEART registry. Int J Cardiol. 2015;185:282-9.

8. Fiedorowicz JG. Depression and cardiovascular disease: an update on how course of illness may influence risk. Curr Psychiatry Rep. 2014;16:492.

9. Compare A, Bigi R, Orrego PS, Proietti R, Grossi E, Steptoe A. Type D personality is associated with the development of stress cardiomyopathy following emotional triggers. Ann Behav Med. 2013;45:299-307.
10. Delmas C, Lairez O, Mulin E, et al. Anxiodepressive disorders and chronic psychological stress are associated with Tako-Tsubo cardiomyopathy- New Physiopathological Hypothesis. Circ J. 2013;77:175-80.

11. Kastaun S, Schwarz NP, Juenemann M, et al. Cortisol awakening and stress response, personality and psychiatric profiles in patients with takotsubo cardiomyopathy. Heart. 2014;100:1786-92.

12. Summers MR, Lennon RJ, Prasad A. Pre-morbid psychiatric and cardiovascular diseases in apical ballooning syndrome (tako-tsubo/stressinduced cardiomyopathy): potential pre-disposing factors? J Am Coll Cardiol. 2010;55:700-1.

13. Pedersen CB. The Danish civil registration system. Scand J Public Health. 2011;39(7 Suppl):22-5.

14. Topp CW, Ostergaard SD, Sondergaard S, Bech P. The WHO-5 Well-Being Index: a systematic review of the literature. Psychother Psychosom. 2015; 84:167-76.

15. Eysenck HJ, Eysenck SBG. Manual of the eysenck personality questionnaire. London: Hodder Stoughton; 1975.

16. Bech P, Jorgensen B, Jeppesen K, Loldrup PD, Vanggaard T. Personality in depression: concordance between clinical assessment and questionnaires. Acta Psychiatr Scand. 1986;74:263-8.

17. Bech P, Rasmussen NA, Olsen LR, Noerholm V, Abildgaard W. The sensitivity and specificity of the Major Depression Inventory, using the Present State Examination as the index of diagnostic validity. J Affect Disord. 2001;66:159-64.

18. Bech P, Bille J, Moller SB, Hellstrom LC, Ostergaard SD. Psychometric validation of the Hopkins Symptom Checklist (SCL-90) subscales for depression, anxiety, and interpersonal sensitivity. J Affect Disord. 2014;160:98-103.

19. Bech P. Clinical psychometrics. 1st ed. Oxford: Wiley Blackwell; 2012.

20. Mokken RJ. Theory and practice of scale analysis. Berlin: Mouton; 1971.

21. Molenaar IWDPSK. User's manual MSP, a program for mokken scale analyses for polytomous items (Version 3.0). Groeningen: ProGAMMA; 1994.

22. Ahtarovski KA, Iversen KK, Christensen TE, et al. Takotsubo cardiomyopathy, a two-stage recovery of left ventricular systolic and diastolic function as determined by cardiac magnetic resonance imaging. Eur Heart J Cardiovasc Imaging. 2014;15:855-62.

23. Nutt DJ. Neurobiological mechanisms in generalized anxiety disorder. J Clin Psychiatry. 2001:62 Suppl 11:22-7.

24. Kalk NJ, Nutt DJ, Lingford-Hughes AR. The role of central noradrenergic dysregulation in anxiety disorders: evidence from clinical studies. J Psychopharmacol. 2011;25:3-16.

\section{Submit your next manuscript to BioMed Central and we will help you at every step:}

- We accept pre-submission inquiries

- Our selector tool helps you to find the most relevant journal

- We provide round the clock customer support

- Convenient online submission

- Thorough peer review

- Inclusion in PubMed and all major indexing services

- Maximum visibility for your research

Submit your manuscript at www.biomedcentral.com/submit
C Biomed Central 